\title{
Neural Pathways Involved in the Hypothalamic Integration of Autonomic Responses
}

\author{
T. L. Powley and W. Laughton \\ Department of Psychological Sciences, Purdue University, West Lafayette, Indiana, USA
}

Summary. Recent mapping studies of hypothalamic and autonomic mechanisms have considerably extended our understanding of the anatomy of this system. The pattern of connections emerging from physiological, anatomical, and histochemical experiments suggests several conclusions about the functional organization of the system as well. Recent evidence supports the idea that the hypothalamic (and other limbic) areas involved in the control of ingestion and metabolism form the rostral pole of a longitudinally and hierarchically organized system that elaborates autonomic responses that influence the energy economy of the animal. Substantially the same pathways are apparently responsible for the modulation of ingestive behavior as well. This circuitry, the "visceromotor system" in Nauta's terminology, seems to weld afferent inputs, particularly those of the gustatory and visceral receptors, into a coordinated integrative control strategy influencing autonomic responses. In addition, the system seems to have unique tissue properties, at least at its two periventricularly located sites of integration with special access to both humorally and ventricularly circulated substrates. These nodes, the basomedial hypothalamus and the vagal complex of the medulla, seem to share similar biochemical specializations reflected in susceptibility to goldthioglucose toxicity, specific insulin binding, and susceptibility to alloxan diabetes.

Key words: Hypothalamus, food intake, metabolism, autonomic nervous system, ingestion, gustatory afferents, visceral afferents.

Understanding of the neural pathways involved in the control of metabolism and ingestion has increased substantially with recent mapping studies.
These studies, both anatomical and electrophysiological, have provided evidence for both previously unrecognized projections and new details about the more classically described central connections. For this review we have focused on selected aspects of that literature, particularly recent mapping work that has identified additional afferent and efferent projections of the hypothalamus. Afferent pathways are reviewed first, and then efferent pathways are examined. Also, in keeping with the orientation of this conference, we have concentrated on the central nervous system (CNS) pathways that are implicated in the control of pancreatic function and ingestive behavior.

The present review also focuses on material relevant to another set of issues. Besides delineating specific connections or pathways, the recent mapping studies taken together reveal a pattern of connections that suggests (at least tentatively) some conclusions about the functional organization of the systems controlling metabolism and ingestion. These functional implications are particularly relevant to the topic of this conference. Material relevant to four of these conclusions is discussed below, but the basic points are briefly summarized here: Firstly, at the level of the hypothalamus, the neural pathways involved in ingestive behavior and those involved in the control of metabolism are tightly articulated, perhaps identical. Secondly, the strong projections from the hypothalamus to the autonomic preganglionic neurons of the medulla and the spinal cord suggest that much of the hypothalamic influence on ingestive behavior (as well as metabolism) may involve the modulation of autonomic responses. Thirdly, the parasympathetic and sympathetic limbs of the autonomic nervous system appear to be controlled in a coordinated, tightly coupled fashion since the key central neural relays involved in autonomic function 
seem to project simultaneously to the preganglionic neurons of both branches of the autonomic nervous system. Fourthly, suggesting that the CNS representation of the autonomic nervous system has special access to the humoral substrates on both the vascular and the ventricular sides, the key integrative loci of the relevant CNS mechanisms lie in unique juxtaposition to specializations of the both the blood-brain barrier and the circumventricular system, and have biochemical specializations seemingly related to their homeostatic roles.

\section{Afferent Inputs Controlling Ingestive Behavior and Metabolism}

Gustatory afferents obviously influence feeding behavior, and they also clearly influence a number of metabolic responses including pancreatic release of insulin by the classic cephalic reflexes [1,2]. Similarly, afferents from the viscera play significant roles in determining food intake $[3,4,5]$ as well as modifying visceral efferent responses through $\mathrm{CNS}$ relays $[6,7]$.

Evidence which has accumulated in the last few years indicates that, as Herrick anticipated years ago [8], these gustatory and visceral afferent fibers interdigitate extensively and have remarkably similar intracranial pathways. In addition, these two sets of afferents both have substantial longitudinal connections with the regions of the hypothalamus and limbic system that have been shown to be involved in the control of caloric intake and insulin release as well as other metabolic responses.

Peripheral gustatory pathways begin at the papillae of the tongue and pharynx, with the lingual branch of the glossopharyngeal nerve, the chorda tympani, and the vagus nerve carrying the sensory input from these receptors [9]. These gustatory afferents terminate primarily in the anterior lateral division of the nucleus of the solitary tract (NST) [10, $11,12,13]$. Peripheral visceral afferents, on the other hand, originate in the mechano- and chemoreceptors of a number of visceral organs, including the stomach, pancreas, and liver $[14,15]$. The parasympathetic afferents ascend in the vagi to the level of the nodose ganglion, where their somata are located, and enter the medulla just dorsal to the inferior olive, synapsing primarily in the medial-caudal pole of the NST [12]. Sympathetic afferents are relayed in the splanchnic nerves to the dorsal root of the spinal cord [9].

Thus, at least for the gustatory afferents relayed over three cranial nerves and the various parasympathetic visceral fibers carried by the vagus, the first synapse in the CNS involves close spatial proximity and perhaps functionally significant overlap in a single relay nucleus. From the NST, second order visceral and gustatory fibers project both rostrally and caudally $[16,17,18]$. Difficulties in obtaining lesions or isotope injections both small enough and accurately placed in one portion or the other of the NST have generally precluded categorical statements concerning differential projections from the gustatory and visceral regions of the nucleus, but evidence now suggests that the projections from these areas are parallel $[18,19]$. Descending connections from the NST project to the motor nuclei of cranial nerves that express skeletal responses and to the preganglionic motor neurons of both branches of the autonomic nervous system, presumably thus providing the oligosynaptic, in theory even disynaptic, pathways that mediate the elemental reflex responses of ingestion and metabolism that are organized in the lower brain stem and spinal cord. Specifically, the NST projects to the hypoglossal, the facial, and (apparently) the trigeminal motor nuclei [18]. In addition, the NST projects to the parasympathetic preganglionic nuclei, the dorsal motor nucleus of the vagus (DMV) [18] and the nucleus ambiguus complex (NA) [16], as well as to the sympathetic preganglionic system, the intermediolateral cell column of the spinal cord (IML) [16]. Finally, the NST also projects to the phrenic motor neurons in the cervical spinal cord and cells in the ventral horn of the thoracic cord [16].

Ascending connections of the NST provide inputs to the more rostral CNS sites involved in the integration of behavioral and metabolic control of homeostasis. Fibers arising from the anterior lateral part of the nucleus have been shown to project to the pontine parabrachial nucleus, also known as the pontine taste area (PTA), where they synapse in the caudal pole [20]. Similarly and in parallel the caudal visceral part of the NST projects to the parabrachial nucleus, where it relays largely in the dorsolateral portion $[19$, 22, 23].

From the PTA, third order axons are relayed rostrally. As the fibre system courses towards the diencephalon, it exhibits a conspicuous bifurcation, sending projections to the classical thalamocortical system and to the hypothalamus and limbic system. The fibers of the thalamocortical system project to the lingual area of the mediobasal thalamus, and from there they project to layer IV of the cortex in an area dorsal to the rhinal sulcus $[21,24]$. The other major projection from the parabrachial nucleus ascends through the subthalamus, giving off collaterals to the lateral hypothalamus, and, after passing through the internal capsule, terminates in the central nucleus of the amygdala and the bed nucleus of 
the stria terminalis. While there is evidence for at least a relative separation of the gustatory and the visceral inputs at both the level of the NST and the level of the PTA, by the level of the hypothalamus and the rest of the limbic system the gustatory and visceral projections clearly converge on common projection fields. Further, at these di- and telencephalic levels there is substantial olfactory, visual, and somatosensory convergence on the same areas [2], suggesting that the areas are involved in the integration of sensory control of responses elaborated by the effectors of the system. In addition to these disynaptic projections relayed by the parabrachial nucleus from the NST to the limbic system, direct (monosynaptic) connections from at least the caudal NST to the paraventricular, dorsomedial and arcuate hypothalamic nuclei as well as to the central nucleus of the amygdala and the bed nucleus of the stria terminalis have been demonstrated [19, 22, 23, 25]. Finally, although the lower brainstem pathways of the afferents carried in the sympathetic trunks are not clearly delineated, there is evidence for splanchnic inputs converging on the same forebrain areas receiving afferents from the NST. Jeanningros and Mei [27] have reported that the vagal and splanchnic afferent projections converge on the same sites in the ventromedial nucleus of the hypothalamus (and see [26]).

Thus afferent information coming from both gustatory and (parasympathetic) visceral receptors is relayed directly from a common brainstem nucleus, the NST, over parallel pathways to skeletal motor neurons and autonomic preganglionic neurons in both the brain stem and spinal cord. This pattern of connections presumably corresponds to the neural circuitry that organizes simple somatic and autonomic reflexes of gustatory and visceral inputs in the brain stem. In addition, the gustatory and visceral divisions of NST also relay projections over the same or parallel pathways to the hypothalamic and limbic (notably amygdaloid) areas classically implicated in the control of ingestion and metabolism. This pattern suggests that these gustatory and visceral afferents modulate hypothalamic and limbic activity in a coordinated manner. The extensive overlap between gustatory and visceral mechnisms would be predicted to lead to significant crosstalk between the input systems, and indeed, there is functional evidence for such interactions $[28,29]$ as well as for extensive convergence of the afferents in the hypothalamus $[27,87]$. As reviewed below, these hypothalamic and limbic system areas receiving gustatory and visceral inputs in turn project back down to the lower brain stem, notably to the autonomic nuclei in the medulla and spinal cord as well as back to the NST and PTA.
This efferent projection profile indicates that the diand tel-encephalic areas involved in ingestion and metabolism have the connections appropriate for modulation of the oligosynaptic reflexes organized and executed by the brainstem and spinal cord. The pattern also suggests that the hypothalamic and other limbic system structures implicated in feeding and metabolic responses have the capability for simultaneous coordination of both the parasympathetic and sympathetic branches of the autonomic nervous system as well as for "editing" or modulating the incoming afferent activity of the NST and PTA.

\section{Descending Hypothalamic Efferents: Physiological Observations}

As is well known, extensive metabolic adjustments are produced by experimental manipulations of the hypothalamus. In general and as described by other papers at this conference, reciprocally organized responses are influenced by manipulations of the medial and the lateral areas of the hypothalamus respectively. Stimulation of the medial portions of the hypothalamus typically leads to an activation of catabolic responses including hyperglycaemia [30, $31,32]$, increases in glycogenolytic enzyme activity $[33,34]$, increased levels of plasma glycerol [35] and non-esterified fatty acids [36], increased plasma glucagon [37], decreased gastric acid secretion [38], and an inhibition of ongoing feeding [39].

Lesions of the ventromedial hypothalamus have described a picture complementary to this, with immediate effects presenting basically the same metabolic profile seen in the chronic VM animal including increased plasma insulin $[40,41]$, normoglycaemia, and increased liver and carcass lipids and glycogen [42], signalling a change in the animal's metabolism to an anabolic state. Behavioral data, of course, show that disabling the ventromedial hypothalamus temporarily by means of local anaesthetics $[43,44]$ results in a rapid feeding response.

Data pertaining to stimulation of the more lateral regions of the hypothalamus, on the other hand, describe a series of anabolic responses, including increased basal [37, 45] and glucose-stimulated [46] insulin release, decreases in hepatic glycogenolytic enzymes [33], increases in hepatic glycogen synthesis [47], and occasionally, increases in gastric acid secretion [48]. Additionally, stimulation of the lateral hypothalamus in freely-behaving animals leads to the well-documented feeding response [49].

Many of these effects elicited from the hypothalamus are clearly mediated by the autonomic 


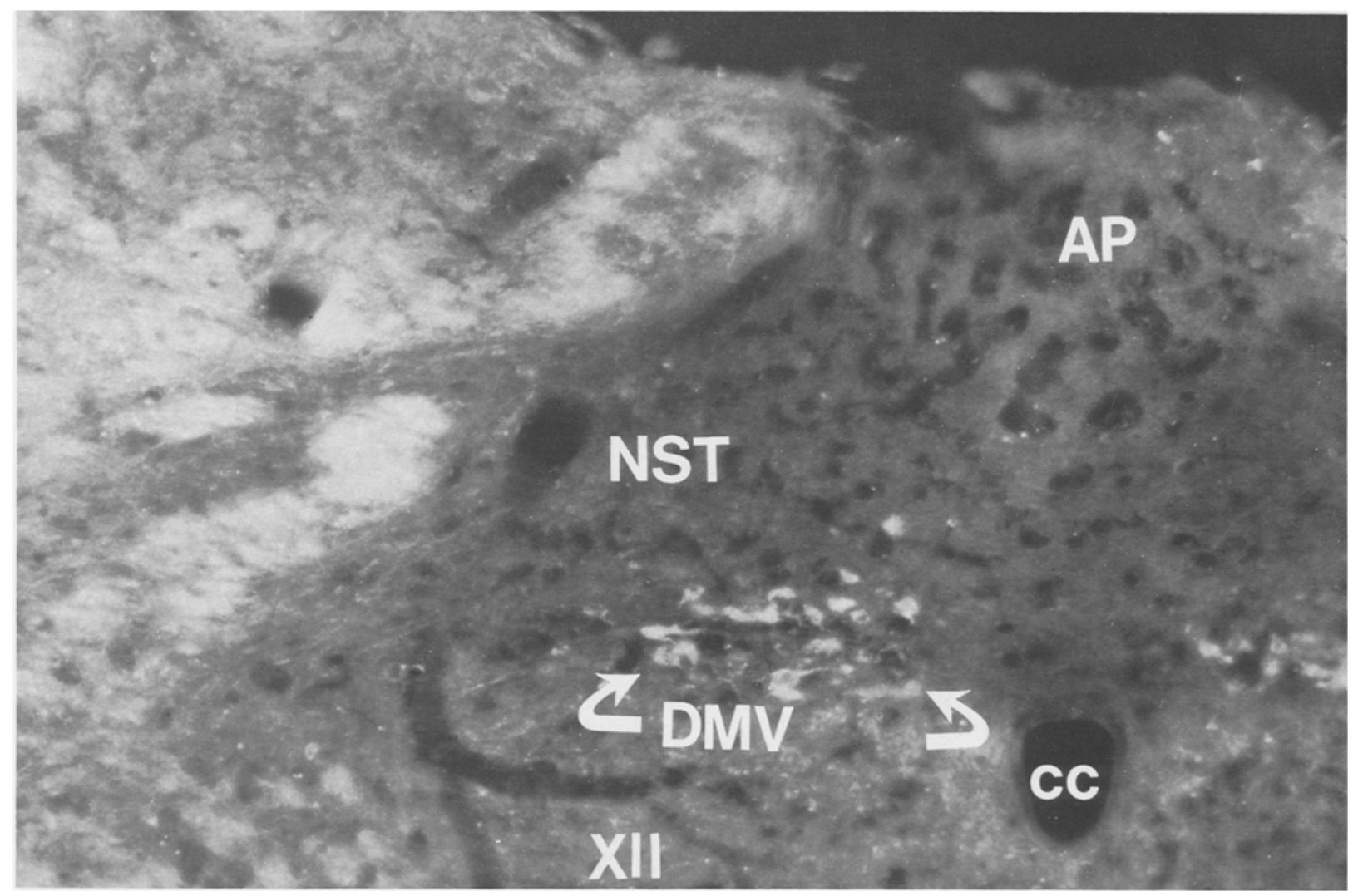

Fig. 1. Low power darkfield photomicrograph of coronal section through the medulla (rat) at the level of the area postrema (AP) showing horseradish peroxidase (HRP)-labelled cells in the dorsal motor nucleus of the vagus (arrows) following injection of $50 \mu 1$ HRP into the pancreas. Smaller injections (down to $2 \mu \mathrm{l}$ ) yield fewer labelled cells. Tetramethylbenzidine technique, neutral red counterstain. AP, area postrema; cc, central canal; DMV, dorsal motor nucleus of the vagus; NST, nucleus of the solitary tract; XII, hypoglossal nucleus

nervous system. The feeding responses and disturbances of ingestive behavior produced by hypothalamic manipulations are potentially major exceptions. However, in the last few years a number of experiments from our laboratory as well as other laboratories have indicated that the feeding responses elicited from the hypothalamus may have essential autonomic components, since both the feeding disturbance of hypothalamic obesity and the feeding behavior elicited by stimulation of the lateral hypothalamus can be blocked or sharply attenuated by subdiaphragmatic vagotomy $[50,51,52,53,54]$. In the case of hypothalamic obesity, the vagal projections to the pancreas seem to be the critical pathway for the expression of the disturbance [51]. In addition, there is evidence that the syndrome associated with destruction of the lateral hypothalamus also involves elements of altered sympathetic and parasympathetic activity $[55,56,57]$. For these different hypothalamic ingestive disturbances, major issues still remain as to the relative importance of the autonomic elements in the various syndromes and as to the balance of altered sympathetic versus altered parasympathetic control in the disturbances $[2,55$, 58]. Regardless of the details of these issues, however, the relevant point is that there is substantial support for the contention that not only the hypothalamic control of metabolic adjustments but also the hypothalamic control of ingestive behavior involves diencephalic modulation of the autonomic preganglionic neurons. The relevance of the point in the present context stems from the fact that the anatomical mapping experiments reviewed below also implicate the hypothalamic areas associated with metabolism and ingestion in an extensively interconnected longitudinal network organized for integration of autonomic responses. On the motor side, long descending projections from the hypothalamus and related limbic areas have been traced directly to the parasympathetic (dorsal motor nucleus of the vagus and NA) and sympathetic (the intermediolateral cell column of the spinal cord) preganglionic motor neurons controlling visceral metabolism. In contrast, recent mapping studies have not revealed such an extensive set of long projections to the skeletal motor systems. 


\section{Descending Efferent Mechanisms: Anatomical Observations}

Early mapping studies employing fibre degeneration methods and Golgi staining techniques suggested that the hypothalamus formed the rostral pole of a "visceromotor" system responsible for the development of autonomic integration [59]. These earlier techniques identified two major efferent outflows of the hypothalamus, the medial forebrain bundle system and the more medial hypothalamic system forming the constituents of the dorsal longitudinal fasciculus of Schutz, connecting virtually all the hypothalamic nuclei implicated in feeding behavior and metabolism with the brain stem regions of the autonomic preganglionic fibers. These classic descending pathways represent polysynaptic projections that make conclusions about the precise sites of termination difficult. Recently, however, the extent of the interaction between the hypothalamus and the autonomic nervous system preganglionics has been underscored by the demonstration of extensive long (that is to say direct, monosynaptic) projections from the hypothalamus to these visceral motor neurons. With high resolution autoradiographic and histochemical procedures, direct projections have been demonstrated from the hypothalamic area, in particular from the paraventricular nucleus, to the NST, DMV, and IML. The strong paraventricular projections are particularly interesting in the context of hypothalamic control of ingestion and metabolism because of recent indications that the paraventricular nuclei or adjacent tissues are important substrates for the classic hypothalamic feeding effects $[87,88]$.

In view of the present emphasis on the neural control of the pancreas, some additional details concerning the localization of parasympathetic preganglionic neurons projection to the organ are relevant before examining the details of the newly identified descending projections from the hypothalamus. In a series of mapping studies with the retrograde tracer enzyme horseradish peroxidase (HRP), we have recently identified the specific location of these vagal preganglionics that project to the pancreas [60] as well as to other viscera (Powley and Laughton, unpublished observations). The cell bodies of the neurons projecting to the pancreas occur in the medial two thirds of the DMV and have their greatest concentration within the nucleus at the rostral-caudal level of the area postrema. Smaller numbers of these cells extend longitudinally in either direction from this central concentration of cells. Figure 1 is a low power photomicrograph of a coronal section containing cells labelled by injection of HRP into the pancreas. The area shown corresponds to the area con- taining the largest number of these labelled cells in the DMV. A smaller number of pancreas-projecting vagal cells are found in a compact cluster in the rostral part of the nucleus ambiguus as well.

In keeping with the hypothalamic organization and the DMV organization just reviewed, recent work indicates that paraventricular nucleus as well as other hypothalamic sites project to NA and DMV, including that part of the DMV containing neurons projecting to the pancreas. Swanson $[61,62]$ has shown a projection of neurophysin-containing fibres from the paraventricular nucleus to the dorsal motor nucleus of the vagus, and Sofroniew and Weindl [63] have demonstrated neurophysin-containing terminals in an area which, judging from their photomicrographs, corresponds to the region of the DMV containining pancreas-projecting neurons (their analysis does not discriminate between neurophysin fibres originating in the paraventricular nucleus and those from the suprachiasmatic nucleus of the hypothalamus however). Monosynaptic projections have been shown from the paraventricular nucleus to the IML of the spinal cord at the midthoracic to upper lumbar levels $[64,65,66,67,68]$. In addition, Ricardo and Koh [23] have demonstrated a projection from the paraventricular nucleus to the caudal (visceral) portion of the NST. The projections to DMV and NST include both oxytocin-neurophysin neurons and vasopressin-neurophysin neurons while those to the IML are primarily oxytocin-neurophysin containing fibers [69]. Finally, the paraventricular nucleus also projects to the medial preoptic area, the bed nucleus of the stria terminalis, amygdala, and median eminence [70].

Efferents from the ventromedial nucleus of the hypothalamus have also been studied recently. Akmayev and coworkers [74] have reported FinkHeimer degeneration in the DMV and nucleus ambiguus after ventromedial hypothalamic lesions associated with obesity. Degeneration has also been identified in the DMV following posterior hypothalamic lesions that presumably encroached on the ventromedial region $[75,76]$. In addition, major projections from the ventromedial nucleus have been noted to the median eminence, bed nucleus of the stria terminalis, and amygdala, among other sites [71, 72]. The ventromedial nucleus also contributes a descending projection which runs dorsocaudally in the periventricular gray to terminate in the mesencephalic and pontine gray matter, forming part of the classic pathway through the dorsal longitudinal fasciculus and connecting (presumably) polysynaptically with cranial nerve nuclei in the medulla [73] such as the DMV, NST, and nucleus ambiguus. 


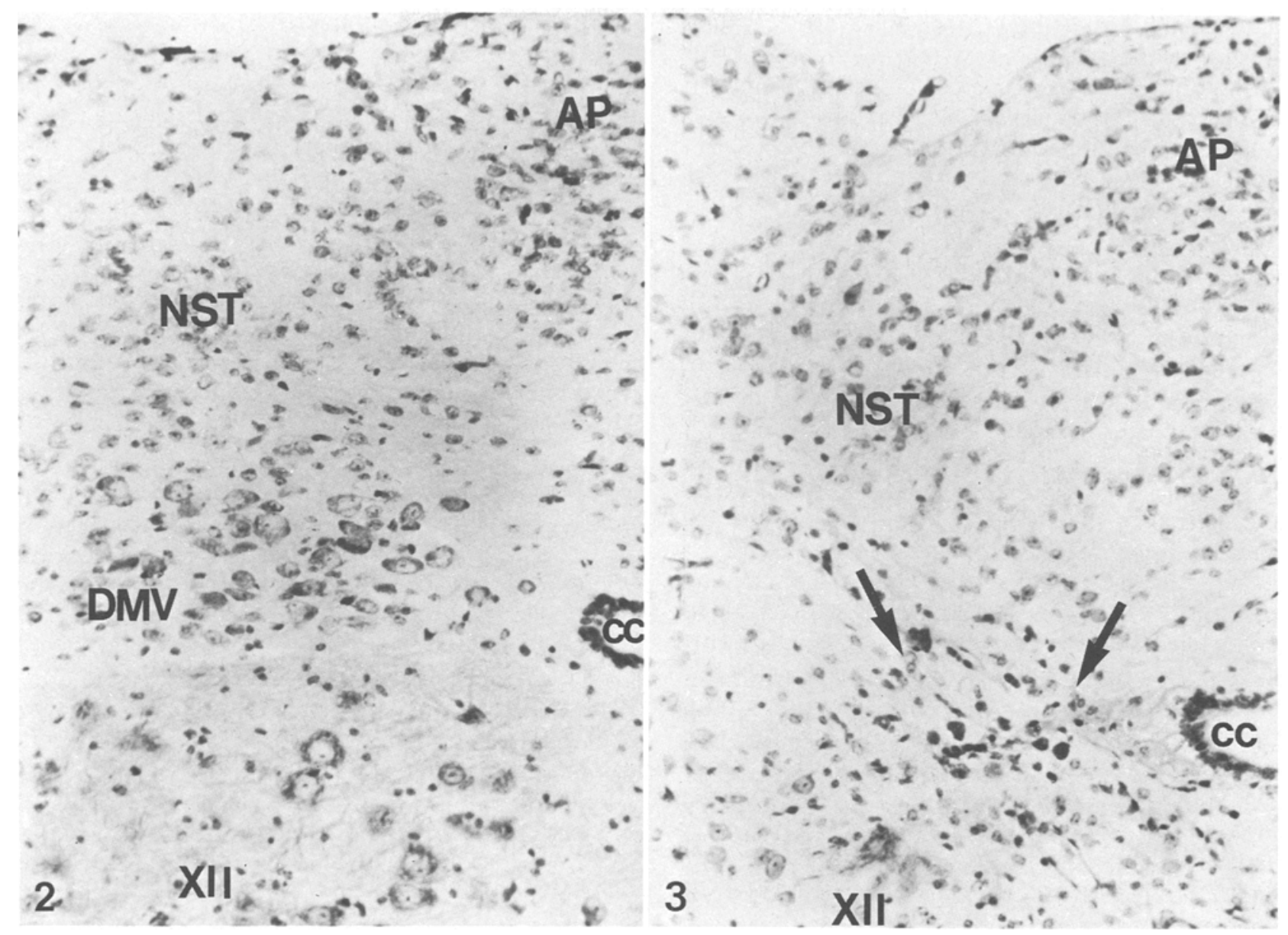

Fig. 2. Lightfield photomicrograph of coronal section through control mouse medulla at the same level as Figure 1, showing medium sized cells in the medial portion of the nucleus of the vagus. Cresyl violet-luxol fast blue. Abbreviations as Figure 1

Fig. 3. Lightfield photomicrograph of coronal section through medulla of goldthioglucose-treated mouse ( $800 \mathrm{mg} / \mathrm{kg})$. Arrows show gliotic scar tissue in the medial portion of the dorsal motor nucleus of the vagus. Cresyl violet-luxol fast blue strain

Relevant projections from the lateral hypothalamus include inputs to the ventromedial nucleus of the hypothalamus as well as those coursing in the dorsal longitudinal fasciculus and reaching the parabrachial nucleus [77]. Recently, Bereiter et al. have demonstrated electrophysiologically that the lateral hypothalamus projects to and can modify the firing of NST neurons driven by chorda tympani branch stimulation [78].

In light of (a) the hypothalamic, pontine, and medullary ascending projections to the amygdala, (b) the well documented influence of limbic regions on food intake [79], and (c) the parallel gustatory and visceral afferent projection to the central nucleus of the amygdala, the descending projection reported by Hopkins and Holstege [80] from the amygdaloid central nucleus to the parabrachial nucleus, locus coeruleus, NST, and DMV also becomes potentially relevant.

Although not anatomical studies per se, two intriguing physiological analyses are particularly relevant to the long longitudinal descending projections from the hypothalamus to the viscera. Both studies are consistent with the suggestion that the autonomic nervous system may be involved in the mediation of the hypothalamic effects on feeding and that these influences, both sympathetic and parasympathetic, remain lateralized and uncrossed from the hypothalamus to the periphery and even the viscera innervated by the preganglionic fibers. Bray and Nishizawa [81] have reported that unilateral lesions of the ventromedial hypothalamic area lead to a slower starvation-produced decline in the weight of retroperitoneal fat pad ipsilateral to the lesion. The 
authors argue that the ventromedial lesions reduces the sympathetic nervous system mediation of fatty acid mobilization from adipose tissue. In a somewhat similar study, England, Marks, Paxinos, and Attens [82] reported that coronal brain hemisections at the level of the caudal pole of the ventromedial nucleus of the hypothalamus selectively reduced stressinduced gastric erosions on the gastric wall innervated by the vagus ipsilateral to the hemisection. Such physiological outcomes are consistent with the anatomical evidence indicating that the descending. projections from the hypothalamus to brain stem ar spinal cord are largely uncrossed [67].

\section{Histochemical Evidence}

In addition to the several physiological experiments and the numerous anatomical analyses suggesting that the hypothalamus and the preganglionic motor neurons of the autonomic nervous system are tightly linked elements of a longitudinally organized control system for energy homeostasis, there exists a third type of evidence. Recent experiments have found that the basomedial hypothalamus and the vagal trigone area of the medulla occupied by the NST and DMV share several tissue specializations, suggesting a commonality of function.

For example, we have found that restricted regions in the DMV and the NST have in common with the ventromedial area of the hypothalamus a special susceptibility to the toxic effects of goldthioglucose. As we have reported [83], goldthioglucose injections that produce basomedial hypothalamic damage also destroy a group of medium sized cells in the medial part of the dorsal motor nucleus at the longitudinal level of the area postrema (the level that contains the largest number of cells projecting to the pancreas). Figure 2 illustrates this level with a photomicrograph of a Nissl-stained coronal section from a control animal. The characteristic cells of the dorsal motor nucleus are labelled. In Figure 3 taken from an animal that had received an injection of goldthioglucose, a gliotic scar can be seen at the site of the dorsal motor nucleus of the vagus. This lesion in the DMV is as well localized and reproducible from animal to animal as is the familiar goldthioglucose lesion of the basomedial hypothalamus. Similarly, there is also some goldthioglucose damage produced in the nucleus of the solitary tract. The lesion is again localized and reproducible; it is confined essentially to the visceral (that is caudal-medial) pole of the NST.

In addition, van Houten and his coworkers [84]; and these proceedings) have shown that essentially the same two sites affected by goldthioglucose, that is the basomedial hypothalamus near the arcuate nu- cleus and the vagal complex near the area postrema, contain high densities of specific insulin binding sites.

Thirdly, in a series of experiments, Akmayev and coworkers $[85,86]$ have shown that the cells of the medial basal hypothalamus, particularly the ventromedial hypothalamic nucleus, and cells of the dorsal motor nucleus of the vagus are selectively and similarly affected by the insulin deficiency associated with alloxan diabetes. The investigators' karyometric analyses demonstrated that the nuclei of cells in the ventromedial nucleus and the DMV were decreased in size, whereas most other cell groups in the hypothalamus and medulla exhibited no changes in nuclear size.

\section{References}

1. Nicolaidis S (1977) Sensory-neuroendocrine reflexes and their enticipatory and optimizing role on metabolism. In: Kare MR, Maller $O$ (eds) The chemical senses and nutrition. Academic Press, New York, p 124-140

2. Powley TL (1977) The ventromedial hypothalamic syndrome, satiety, and a cephalic phase hypothesis. Psychol Rev 84: 89-126

3. Novin D, VanderWeele DA (1977) Visceral involvement in feeding: there is more to feeding than the hypothalamus. In: Sprague JM, Epstein AN (eds) Progress in psychobiology and physiological psychology, vol 7. Academic Press, New York, p 193-241

4. Sawchenko PE, Friedman MI (1979) Sensory functions of the liver - a review. Am J Physiol 236: R5-R20

5. Wyrwicka W (1969) Sensory regulation of food intake. Physiol Behav 4: 853-858

6. Sakaguchi T, Yamaguchi K (1979) The effect of electrical stimulation of the hepatic branch of the vagus nerve on the secretion of gastric acid in the rat. Neurosci Lett 13: 25-28

7. Sakaguchi T, Yamaguchi K (1979) Effects of electrical stimulation of the hepatic vagus nerve on the plasma insulin concentration in the rat. Brain Res 164: 314-316

8. Herrick CJ (1944) The fasciculus solitarius and its connections in amphibians and fishes. J Comp Neurol 81: 307-331

9. Goss CM (ed) (1975) Gray's anatomy of the human body. Lea and Febiger, Philadelphia, p 1038

10. Astrom KE (1953) On the central course of afferent fibers in the trigeminal, glossopharyngeal, and vagal nerves and their nuclei in the mouse. Acta Physiol Scand 29 [Suppl 106]: 209-320

11. Kerr FWL (1962) Facial, vagal, and glossopharyngeal nerves in the cat. Afferent connections. Arch Neurol 6: 264-281

12. Rhoton AL Jr, O'Leary JL, Ferguson JP (1966) The trigeminal, facial, vagal, and glossopharyngeal nerves in the monkey. Arch Neurol 14: 530-540

13. Torvik A (1956) Afferent connections to the sensory trigeminal nuclei, the nucleus of the solitary tract and adjacent structures. An experimental study in the rat. J Comp Neurol 106: 51-141

14. Tsai TL (1958) A histological study of the sensory nerves in the liver. Acta Neuroveg 17: 354-385

15. Paintal AS (1973) Vagal sensory receptors and their relex arcs. Physiol Rev 53: 159-227

16. Loewy AD, Burton H (1978) Nuclei of the solitary tract: efferent projections to the lower brain stem and spinal cord of the cat. J Comp Neurol 181: 421-450

17. Cottle MKW, Calaresu FR (1975) Projections from the nu- 
cleus and tractus solitarius in the cat. J Comp Neurol 161: $143-158$

18. Norgen, R (1978) Projections from the nucleus of the solitary tract in the rat. Neuroscience 3: 207-218

19. Koh ET, Ricardo JA (1978) Afferents and efferents of the parabrachial region in the rat: evidence for parallel ascending gustatory versus visceroceptive systems arising from the nucleus of the solitary tract. Anat Rec 190: 449

20. Norgren R, Leonard CM (1973) Ascending central gustatory pathways. J Comp Neurol 150: 217-238

21. Norgen R, Wolf G (1975) Projections of thalamic gustatory and lingual areas in the rat. Brain Res 92: 123-129

22. Ricardo J, Koh ET (1978) Anatomical evidence of direct projections from the nucleus of the solitary tract to the hypothalamus, amygdala, and other forebrain structures in the rat. Brain Res 153: 1-26

23. Koh ET, Ricardo J (1979) Connections of hypothalamic neurosecretory nuclei with sensory structures in the brain stem of the rat. Neurosci Abstr 5: 450

24. Nomura $S$, Mizuno $N$, Itoh $K$, Matsuda $K$, Sugimoto $T$, Nakamura Y (1979) Localization of parabrachial neurons projecting to the thalamus or the amygdala in the cat using horseradish peroxidase. Exp Neurol 64: 375-385

25. Kogure S, Onoda N, Takagi SF (1978) Olfactory responses of lateral hypothalamic neurons to stomach distension in unanesthetized rabbits. Proc Jpn Acad 54B: 478-483

26. Chaput M, Holley A (1976) Olfactory bulb responsiveness to food odour during stomach distension in the rat. Chem Senses Flavor 2: 189-201

27. Jeanningros R, Mei N (1980) Vagal and splanchnic effects at the level of the ventromedian nucleus of the hypothalamus (VMH) in the cat. Brain Res 185: 239-251

28. Glenn JF, Erikson RP (1976) Gastric modulation of gustatory afferent activity. Physiol Behav 16: 561-568

29. Hellekant $G$ (1971) The effect of stomach distension on the efferent activity in the chorda tympani nerve of the rat. Acta Physiol Scand 83: 527-531

30. Zukoski CF, Lee HM, Hume DM (1963) Effect of hypothalamic stimulation on gastric secretion and adrenal function in the dog. J Surg Res 3: 301-306

31. Barkai A, Allweis C (1972) Effect of electrical stimulation of the hypothalamus on plasma levels of free fatty acids and glucose in rats. Metabolism 21: 921-927

32. Frohman LA, Bernardis LL (1971) Effect of hypothalamic stimulation on plasma glucose, insulin, and glucagon levels. Am J Physiol 221: 1596-1603

33. Shimazu T, Ogasawara S (1975) Effects of hypothalamic stimulation on gluconeogenesis and glycolysis in rat liver. Am J Physiol 228: 1787-1793

34. Matsushita H, Shimazu T (1980) Chemical coding of the hypothalamic neurons in metabolic control. II. Norepinephrine-sensitive neurons and glycogen breakdown in liver. Brain Res 183: 79-87

35. Kumon A, Takahashi A, Hara T, Shimazu T (1976) Mechanism of lipolysis induced by electrical stimulation of the hypothalamus in rabbits. J Lipid Res 17: 551-558

36. Texeira VL, Atunes-Rodriguez J, Migliorini RH (1975) Evidence for centers in the central nervous system that selectively regulate fat mobilization in the rat. J Lipid Res 14: 672-677

37. deJong A, Strubbe JH, Steffens AB (1977) Hypothalamic influence on insulin and glucagon release in the rat. Am J Physiol 233: E380-E388

38. Misher A, Brooks FP (1966) Electrical stimulation of the hypothalamus and gastric secretion in the albino rat. Am J Physiol 211: 403-405

39. Hoebel BG, Teitlebaum P (1962) Hypothalamic control of feeding and selfstimulation. Science $135: 375-377$
40. Rohner F, Dufour A-C, Karakash AC, LeMarchand X, Ruf KB, Jeanrenaud B (1977) Immediate effects of lesions of the ventromedial hypothalamic area upon glucose-induced insulin secretion in anesthetized rats. Diabetologia 13: 239-242

41. Hustvedt B-E, Lovo A (1972) Correlation between hyperinsulinemia and hyperphagia in rats with ventromedial hypothalamic lesions. Acta Physiol Scand 84: 29--33

42. MacKenzie RG, Bernardis LL, Goldman JK (1979) Early metabolic effects of ventromedial hypothalarnic lesions. Neurosci Abstr 5: 220

43. Berthoud HR, Jeanrenaud B (1979) Changes of insulinemia, glycemia, and feeding behaviour induced by VMH-procainization in the rat. Brain Res 174: 184-187

44. Maes $H$ (1979) Feeding, scanning and photophobia after local injections of pentobarbital or noradrenaline into the ventromedial hypothalamus. Behav Proc 4: 239-252

45. Stulnikov V (1973) Role of the lateral and ventro-medial portions of the hypothalamus in regulation of insulin secretion. Byull Eksp Biol Med 76: 3-7

46. Curry DL, Joy RM (1974) Direct CNS modulation of insulin secretion. Endocr Res Commun 1: 229-237

47. Matsushita H, Ishikawa K, Shimazu T (1979) Chemical coding of the hypothalamic neurons in metabolic control. I. Acetycholine-sensitive neurones and glycogen synthesis in liver. Brain Res 163: 253-261

48. Carmona A, Slagen J (1973) Effects of chemical stimulation of the hypothalamus upon gastric secretion. Physiol Behav 10: 657-661

49. Powley TL, MacFarlane BA, Markell MS, Opsahl CA (1978) Different effects of vagotomy and atropine on hypothalamic stimulation induced feeding. Behav Biol 23: 306-325

50. Powley TL, Opsahl CA (1974) Ventromedial hypothalamic obesity abolished by subdiaphragmatic vagotomy. Am J Physiol 226: 25-33

51. Sawchenko PE (1979) Vagal mediation of hypothalamic hyperphagia and obesity. Doctoral dissertation, University of Massachusetts

52. Rowland N, Engle DJ (1978) Hypothalamic hyperphagia prevented by prior subdiapharagmatic vagotomy: insulin hyperphagia is unaffected. Physiol Behav 21: 685-689

53. Cox JE (1980) The role of autonomic reflexes in the ventromedial hypothalamic syndrome. Doctoral dissertation, Yale University

54. Inoue S, Bray GA (1977) The effects of subdiaphragmatic vagotomy in rats with ventromedial hypothalamic lesions. Endocrinology 100: 108-114

55. Powley TL, Opsahl CA (1976) Autonomic components of the hypothalamic feeding syndromes. In: Novin DA, Wyrwicka W, Bray GA (eds) Hunger: Basic mechanisms and clinical implications. Raven Press, New York p 313-326

56. Opsahl CA (1977) Sympathetic nervous system involvement in the lateral hypothalamic lesion syndrome. Am $\mathbf{J}$ Physiol 232: R128-R136

57. Opsahl CA, Powley TL (1977) Body weight and gastric acid secretion in rats with subdiaphragmatic vagotomy and lateral hypothalamic lesions. J Comp Physiol Psychol 91: 1.284-1296

58. Inoue S, Mullen Y, Bray GA (1979) Mechanism of hyperinsulinemia in ventromedial hypothalamic (VMH) obesity in rats. Proceedings of the Endocrine Society

59. Nauta WJH (1972) The central visceromotor system: a general survey. In: Hockman $\mathrm{CH}$ (ed) Limbic system mechanisms and autonomic functions. CC Thomas, Springfield IL, p 21-38

60. Laughton WL, Powley TL (1979) Four central nervous system sites project to the pancreas. Neurosci Abstr 5: 46

61. Swanson LW (1978) Extrahypophseal oxytocin-containing pathways in the brain and spinal cord of the rat and monkey. Neurosci Abstr 4: 415 
62. Swanson LW (1977) Immunohistochemical evidence for a neurophysin-containing autonomic pathway arising in the paraventricular nucleus of the hypothalamus. Brain Res 128 : 346-353

63. Sofroniew MV, Weindl A (1978) Projections from the parvocellular vasopressin- and neurophysin-containing neurons of the suprachiasmatic nucleus. Am J Anat 153: 391-430

64. Buijs, RM (1978) Intra- and extrahypothalamic vasopressin and oxytocin pathways in the rat. Pathways to the limbic system, medulla oblongata, and spinal cord. Call Tissue Res 192: 423-435

65. Ono T, Nishino H, Sasaka K, Muramoto K, Yano I, Simpson A (1978) Paraventricular nucleus connections to spinal cord and pituitary. Neurosci Lett 10: 141-146

66. Hoyosa Y, Matsushita M (1979) Identification and distribution of the spinal and hypophyseal projection neurons in the paraventricular nucleus of the rat. A light and electron microscopic study with the horseradish peroxidase method. Exp Brain Res 35: 315-331

67. Saper CB, Loewy AD, Swanson LW, Cowan WM (1976) Direct hypothalamoautonomic connections. Brain Res 117: 305-312

68. Hancock MB (1976) Cells of origin of hypothalamo-spinal projections in the rat. Neurosci Lett 3: 179-184

69. Nilaver G, Zimmermann EA, Wilkins J, Michaels J, Hoffman D, Silverman A (1980) Magnocellular hypothalamic projections to the lower brain stem and spinal cord of the rat. Neuroendocrinology $30: 150-158$

70. Conrad LCA, Pfaff DW (1976) Efferents from medial basal forebrain and hypothalamus in the rat. II. An autoradiographic study of the anterior hypothalamus. J Comp Neurol 196: 221-262

71. Krieger MS, Conrad LCA, Pfaff DW (1979) An autoradiographic study of the efferent connections of the ventromedial nucleus of the hypothalamus. J Comp Neurol 183: 785-816

72. Saper CB, Swanson LW, Cowan WM (1976) The efferent connections of the ventromedial nucleus of the hypothalamus of the rat. J Comp Neurol 169: 409-442

73. Ban T (1964) The hypothalamus, expecially on its fiber connections, and the septopreopico-hypothalamic system. Med J Osaka Univ 15: 1-83

74. Akmayev IG, Rabkina AE (1978) CNS-endocrine pancreas system. IV. Evidence for existence of a direct hypothalamicvagal descending pathway. Endocrinology $71 ; 175-182$

75. Cheatham ML, Matzke HA (1966) Descending hypothalamic medullary pathways in the cat. J Comp Neurol 127: 369-380

76. Ciriello J, Calaresu FR (1977) Descending hypothalamic pathways with cardiovascular function in the cat: A silver impregnation study. Exp Neurol 57: 561-580
77. Saper CB, Swanson LW, Cowan WM (1979) An autoradiographic study of the efferent connections of the lateral hypothalamic area in the rat. J Comp Neurol 183: 689-706

78. Bereiter D, Berthoud HR, Jeanrenaud B (1980) Hypothalamic input to brain stem neurons responsive to oropharyngeal stimulation. Exp Brain Res 39: 33-39

79. Morgane PJ (1962) Hypothalamic and rhinencephalic mechanisms in the regulation of calorie intake. Proc XXIII International Physiol Cong, Leiden, Netherlands, p 670-676

80. Hopkins DA, Holstege G (1978) Amaygdaloid projections to the mesencephalon, pons, and medulla oblongata in the cat. Exp Brain Res 32: 529-547

81. Bray GA, Nishizawa Y (1978) Ventromedial hypothalamus modulates fat mobilisation during fasting. Nature 274: 900-902

82. England AS, Marks PC, Paxinos G, Atrens DM (1979) Brain hemisections induce asymmetric gastric ulceration. Physiol Behav 23: 513-517

83. Powley TL, Low EL, Plocher TA (1977) Goldthioglucose destroys a specific population of neurons within the dorsal motor nucleus of the vagus. Proc VI International Conf Physil, Food and Fluid Intake, Paris, France

84. van Houten M, Posner BI, Kopriwa BM, Brawer JR (1979) Insulin binding sites in rat brain: In vivo localization to circumventricular organs by quantitative autoradiography. Endocrinology 105: 666-673

85. Akmayev IG, Rabkina AE (1976) CNS-endocrine pancreas system. I. The hypothalamus response to insulin deficiency. Endocrinology 68: 211-220

86. Akmayev IG, Rabkina AE (1976) CNS-endocrine pancreas system. II. Response of dorsal nucleus of the vagus nerves to insulin deficiency. Endocrinology 68: 221-225

87. Beklavadzhyan OG, Adamyan EA, Avetisyan EA, Bagdasaryan KG (1977) Convergence of visceral afferent impulses on hypothalamic neurons during vagal and splanchnic stimulation. Nierofiziologiya 9: 126-131

88. Eng R, Gold RM, Nunez A (1979) Elemental hypothalamic obesity after discrete lesions of the paraventricular nucleus. Neurosci Abstr 5: 216

89. Leibowitz SF (1979) Functional and anatomical studies of noradrenergic system of the paraventricular hypothalamus that controls feeding behavior. Neurosci Abstr 5: 220

Terry L. Powley

Department of Psychological Sciences

Purdue University

West Lafayette, IN 47907

USA

\section{Discussion after Powley's Presentation}

Oomura: Have you tried your experiments with goldthioglucose in the rat?

Powley: As you know, goldthioglucose in nontoxic doses has traditionally been thought to be ineffective in the rat. There are ways around the problem if the drug is put directly in the brain, and there are other ways to make neurochemical lesions which selectively map themselves onto these same areas. We've recently begun trying some of these other compounds in the rat.
Sclafani: What happens to vagal activity in goldthioglucose-lesioned animals?

Powley: No one knows. One might presume that the lesion is like a vagotomy of sorts. However, the animals do become obese and have hyperinsulinaemia. On the other hand, I should point out that the goldthioglucose does not kill all of the cells in the dorsal motor nucleus of the vagus. So I would have to guess that goldthioglucose causes an incomplete vagotomy in these animals but that their vagal activity ought to be investigated. 
Sclafani: Do these animals get fat if they are not allowed to overeat?

Powley: Pair-feeding has not been done to any great extent with these animals. There is good evidence that gold thioglucose animals fatten without overeating in a neutral thermal environment.

Woods: When you inject HRP into the pancreas, how long does it take to get into these brainstem cells?

Powley: We haven't done a complete time series on these animals. People ordinarily wait from 48 to $72 \mathrm{~h}$. In the studies I reported, we waited $48 \mathrm{~h}$. The enzyme is thought to be sufficiently stable that one has considerable latitude in this regard.

Bray: You've done some elegant work on the parasympathetic side of this system. For those of us interested in the sympathetic side, how would you go about doing mapping studies since the peripheral neuroanatomy is so different?

Powley: It's clear that HRP and other neuroanatomical procedures won't easily provide as much information because of the reasons you allude to, so I expect that considerable more neurophysiological work is going to have to be done in conjunction with anatomical investigations.

Van Houten: Your work is very impressive. Let me first point out that the areas which you have described as projecting to the pancreas appear to be precisely those areas where we have found evidence for insulin receptors, suggesting a direct feedback loop to those neurons which influence secretion.

Powley: Yes, I am very excited by that prospect.

Van Houten: We have also used goldthioglucose in some experiments and have found that it reduces insulin binding in both the ventral hypothalamus and the area postrema. In fact, in some animals, specific binding sites are essentially absent. Have you ever tried monosodium glutamate (MSG)?

Powley: We have used some biperperidyl mustard and obtained similar lesions, but we haven't given MSG.

Berthoud: Have you tried to lesion these areas electrolytically in rats?

Powley: We have been developing a technique for this and have spent considerable time working out coordinates and other parameters. The longitudinal nature of the cellular column requires a multiple-lesion technique. Now that we seem to have the procedure, we intend to look at the metabolic effects of the lesions.

Sclafani: Given the bloodflow of the hindbrain, would it be possible to inject goldthioglucose so it lesioned only the hindbrain sites and not the hypothalamus?

Powley: You could try it, but it would be very complicated in the mouse where most of the research has been done. It would probably require the simultaneous injection of the drug into the basilar artery and ligation of others.
Porte: I'm confused as to the nature of the goldthioglucose lesion. What actually dies?

Powley: Under the light microscope, it certainly appears that the cell bodies, i.e. the neurons in the NTS and dorsal motor nucleus, are dying. There presumably are other cellular and vascular effects which can't be see at this level, but the bottom line seems to be that neuronal death occurs in the medial pole of the NTS and dorsal motor nucleus.

Porte: Is the pattern similar in the hypothalamus?

Nicolaidis: There is evidence from Mayer's group (Exp Neurol (1969) 25: 410-415) that blood vessels are actually the first tissues to be destroyed in the hypothalamus and that neuronal death is secondary to reduced blood supply. The extent of the lesions is also very large.

Powley: I'd like to qualify that. There's no doubt that goldthioglucose is a dirty compound. After it was initially used, a lot of experiments appeared which emphasized how dirty it really is. For example, larger doses clearly cause damage in a number of areas of the brain. Some of this work suggested that gold thioglucose is effective solely because it produces inflammation and distension of specialized perivascular spaces. However, that work was not exhaustive. There's been a recent report by Kataoka and coworkers (Brain Res Bull (1978) 3: 257-263) suggesting the compound has two sites of action: the vascular endothelium and a membrane binding site as well. Further, mapping studies indicate that at lower doses the gold thioglucose damage is restricted to the hypothalamus and medulla.

Porte: So what doses are the best to use?

Powley: In the $\mathrm{C} 57$ black mouse, the smallest effective dose is around $400 \mathrm{mg} / \mathrm{kg}$. At doses up to $800 \mathrm{mg} / \mathrm{kg}$, the lesions are still rather restricted. Above that, there's substantial "non-specific" damage as well. In other strains of mice, the scale is shifted.

Van Houten: In our studies where we were looking at the area postrema and the ventral hypothalamus, we were concerned about these problems. We gave $400 \mathrm{mg} / \mathrm{kg}$ and waited 6 months to allow the blood vessels to be restored before doing the autoradiographic studies. Electron microscopy of the GTG-lesioned arcuate nucleus revealed numerous healthy neurons in this area. However, axonal growth cones were abnormally abundant suggesting that connections had been damaged.

Porte: As I recall your slides, the cells in the area postrema where you found insulin binding is not exactly the same area destroyed by goldthioglucose in Dr. Powley's experiments.

Van Houten: That's true, but in the work I'm presently doing with Dr. Woods and Dr. Baskin in Seattle, we put the labelled insulin directly into the ventricles. In that instance, the insulin-concentrating neurons seem to form a necklace around the area postrema, and these cells are at least close to, if not the same cells, as those described by Dr. Powley. 